\title{
Introducción del Operario Humano en el Ciclo de Automatización de Procesos Mediante la Guía GEMMA
}

\author{
Pere Ponsa ${ }^{(1)}$, Ramón Vilanova ${ }^{(2)}$ y Marta Díaz $^{(3)}$ \\ Universidad Politécnica de Cataluña, (1) Departamento de Ingeniería de Sistemas, Automática e \\ Informática Industrial, (3) Departamento de Organización de Empresas, Av. Víctor Balaguer s/n, \\ 08800 Vilanova i la Geltrú-España (e-mail: pedro.ponsa@upc.edu; marta.diaz@upc.edu) \\ (2) Universidad Autónoma de Barcelona, Departamento de Telecomunicación e Ingeniería de \\ Sistemas, ETSE, 08913 Bellaterra-España (ramon.vilanova@uab.cat)
}

\section{Resumen}

En este artículo, se presenta el estudio concreto de la intervención de operarios en sistemas automatizados a los que se ha aplicado la metodología llamada guía GEMMA (Estudio de Modos de Marchas y Paros). Siendo esta guía, una buena aproximación metodológica para resolver problemas complejos de automatización. Aunque su uso no es extenso, constituye una guía rigurosa que permite abordar la transición de los ejemplos académicos a las especificaciones industriales. La inclusión del operario humano, así como la comunicación con los dispositivos de automatización, necesita de guías metodológicas que puedan aunar la estructuración propuesta en la guía GEMMA con diversos conceptos extraídos de la ergonomía cognitiva y la interacción persona-ordenador. Se concluye que la guía GEMMA presenta una metodología integradora concebida para clarificar el rol del operario humano en el ciclo de automatización de sistemas complejos con aplicación en el control y supervisión de procesos industriales.

Palabras claves: control, supervisión, automatización, interacción persona-ordenador, guía GEMMA

\section{GEMMA Guide Approach for the Introduction of the Human Operator into the Automation Cycle}

\begin{abstract}
The use of the design guide to start and stop modes, GEMMA guide, is known as a good approach to deal with the task of solving complex automation problems. Even not widely used, it constitutes a really good methodological approach that allows to smooth the transition from academical examples to industrial settings. The purpose of this paper is to show an additional advantage of the GEMMA guide that should motivate its use as well as introduce the consideration of the human operator as an integral part of the automation procedure. The inclusion of the human operator as well as his communication with the automation device needs some guidelines that can be drawn from joining the GEMMA structured approach with some concepts borrowed from cognitive ergonomic theory and human-computer interaction.
\end{abstract}

Keywords: control, supervision, automation, human-computer interaction, GEMMA guide 


\title{
INTRODUCCIÓN
}

El diseño de sistemas de producción complejos requiere de nuevas herramientas y metodologías. Desde el punto de vista académico, se ha contribuido mediante el uso de las redes de Petri (Petri Nets-PN), sobre problemas diversos de modelado de procesos en automatización (Kontogiannis, 2005). Uno de los aspectos no resueltos mediante el formalismo de las redes de Petri, es la introducción de las tareas humanas en el modelo descrito en forma de estados y transiciones. En todo problema de automatización, la secuencia de operaciones a realizar por la máquina tan solo es una parte. Es necesario analizar como el operario humano accede y participa también en el ciclo de operaciones básicas. De igual modo, es importante poner de manifiesto cómo se lleva a cabo la interacción entre el operario humano y el controlador lógico programable (PLC), encargados ambos de compartir las tareas de control de las operaciones. Para aunar automatización y tareas humanas es necesario acercar las disciplinas automatización industrial e interacción persona-ordenador (Armengol et al., 2000; Cañas, 2004; Cavaría, 2007; Mondelo, 2001; Carver y Turoff, 2007; Ponsa y Díaz, 2007). Antes de proceder a estudios de campo o a la aplicación de métodos etnográficos para la obtención de la elicitación del conocimiento es menester empezar por la investigación en el laboratorio mediante estudios prácticos de escenarios en los que aplicar las técnicas de ergonomía y usabilidad.

En este artículo se presenta el estudio concreto de la intervención de operarios en sistemas automatizados a los que se ha aplicado la metodología llamada guía GEMMA (ADEPA, 1981), detallada en las dos siguientes secciones (La guía GEMMA, Diseño estructurado). Siguiendo el desarrollo de la metodología presentada, la sección llamada Diseño de panel industrial muestra cómo la ergonomía puede aplicarse al diseño de paneles de mando en automatización industrial. El centro docente de la Escuela Politécnica Superior de Ingeniería de Vilanova i la Geltrú (EPSEVG), dispone de un Laboratorio docente de Sistemas de Producción en el que se reproduce de forma didáctica las tareas de los operarios de planta (mantenimiento, automatización, vigilancia del proceso controlado, intervención del operario mediante panel de mando, supervisión mediante herramientas gráficas comerciales, seguridad del sistema persona-máquina), en un sistema de fabricación académico. La sección citada muestra las tareas llevadas a cabo por ingenieros técnicos sobre un panel de mando didáctico en el que se presentan indicaciones ergonómicas para la mejora del panel utilizado. En la sección de conclusiones se valora los resultados obtenidos y se plantean las líneas siguientes de trabajo a acometer.

\section{LA GUÍA GEMMA}

La guía GEMMA procede de los trabajos llevados a cabo durante dos años por la Agencia Nacional Francesa para el Desarrollo de la Producción Aplicada a la Industria (ADEPA, 1981). Las siglas GEMMA (Guide d'Etude des Modes de Marches et d'Arrets), significan Guía de Estudio de los Modos de Marcha y Paro. En el contexto de su creación, a lo largo del año 1993, se concibe para que esté en consonancia con las normas de seguridad de la Unión Europea. Bajo la norma nacional francesa UTE C 03-191, se complementa con la representación GRAFCET (Grafo de estados y transiciones), (AFCET-ADEPA, 1995) y pretende dar cabida a una metodología que incluya los modos de marcha y paro del control secuencial, el funcionamiento correcto del proceso controlado junto al funcionamiento deteriorado ante anomalías, e incluso el tratamiento de situaciones de emergencia en previsión de posibles daños humanos o materiales (Garcia, 2004). La metodología presente en la guía GEMMA (Ponsa y Vilanova, 2005) consiste en un conjunto de fases:

\author{
- Automatización \\ - Control Supervisor (Supervisory control) \\ - Interacción \\ - Implantación \\ - Test
}

La aproximación clásica se focaliza en los aspectos de automatización, ya que los futuros operarios han cursado estudios reglados en el área de ingeniería y son expertos en controladores lógicos programables PLC, neumática, robótica y sensórica (Fig. 1). 


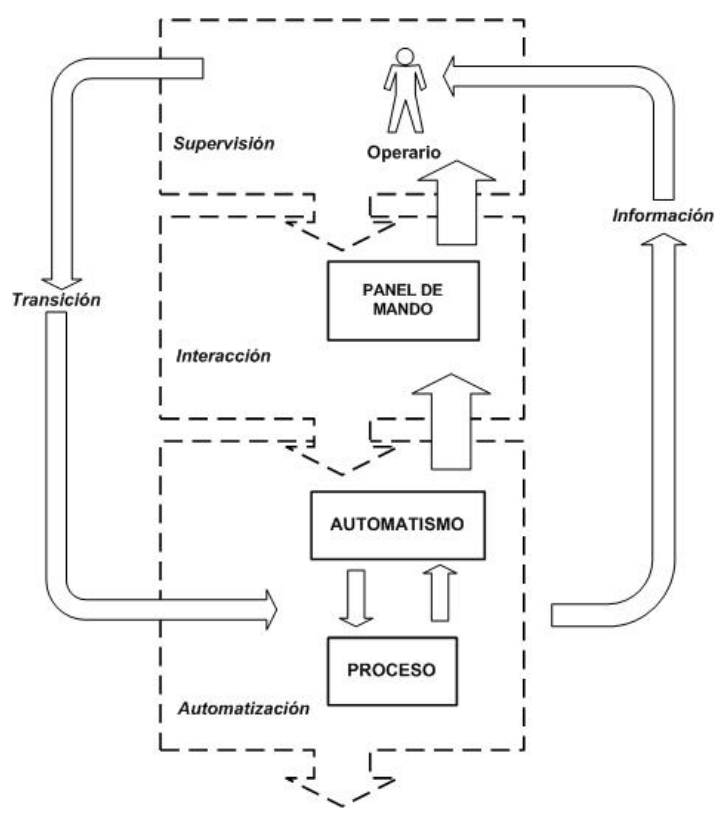

Fig. 1: Interacción persona-máquina en automatización industrial

En la fase de control supervisor, se pretende remarcar el rol de vigilancia del proceso controlado por parte del operario humano (Català et al., 2000; Petersen, 2004; Ponsa y Català, 1999). En la fase de interacción, el operario interviene mediante un panel de mando. Es importante plantear el diseño del panel de mando, en función de las acciones físicas del operario sobre dispositivos y la recepción de señales informativas visuales o acústicas (Oncins, 2007). En la fase de implantación se procede a la programación de la guía GEMMA dentro del automatismo (controlador lógico o PLC). En la fase de test el operario, se puede verificar el correcto funcionamiento, e introducir mejoras de forma iterativa en cada una de las fases mencionadas. La guía GEMMA se presenta como una lista gráfica de modos que permiten al operario humano definir desde el inicio todas las operaciones y sus consecuencias sobre la máquina (Kontogiannis, 1996). La guía GEMMA parte de diversos conceptos básicos:

- condiciones previas de conexión de energía a la máquina

- procedimientos de marcha y paro de la máquina (control manual y/o control automático)

- procedimientos de funcionamiento de la máquina (funcionamiento normal o anómalo)

- procedimientos de fallos y/o defectos de la máquina (tratamiento de situaciones de emergencia, reconducción de la máquina a situaciones seguras de funcionamiento)

A partir de estas ideas, la guía GEMMA se crea como una representación gráfica del conjunto de conceptos descritos en forma de modos operativos (asociados en familias de procedimientos A, F, D) y transiciones entre modos. A continuación se describen los modos más relevantes.

PZ1: Puesta del control sin energía. Cuando el automatismo se queda sin energía, este estado se ocupa de los procedimientos a realizar para garantizar la seguridad.

F1: Producción normal. Estado en el que la máquina está produciendo normalmente, realizando la tarea para la que está concebida. En su interior, se encuentra el algoritmo básico, expresado habitualmente como GRAFCET de producción o GRAFCET de base.

F4: Marcha de verificación sin orden. Permite certificar movimientos individuales del ciclo, sin respetar el orden habitual (usualmente, en vacío). Es asimilable a la intervención del operario en la forma de control manual.

A1: Parada en el estado inicial. Estado inicial de reposo de la guía GEMMA. Se suele corresponder con el estado inicial del GRAFCET. 
A2: Parada a final de ciclo. Cuando se solicita este paro, la máquina debe completar el ciclo y luego detenerse en el estado inicial. En caso contrario, no se produce interrupción alguna y el ciclo se repite automáticamente.

A5: Preparación para la Puesta en Marcha después de un Fallo. En este estado, deben efectuarse las operaciones necesarias para una nueva puesta en marcha después de un fallo/defecto (vaciado, limpieza, reposición de producto, etc.). Se trata de reconfigurar el sistema y, una vez concluida la preparación, habitualmente se asocia a un pulsador con la finalidad de rearme.

D1: Parada de emergencia (o parada para asegurar la seguridad). Estado al que evoluciona el sistema después de accionar el paro de emergencia. Debe preverse un cese de actividades lo más rápido posible y las actuaciones necesarias para limitar las consecuencias del paro (tanto en la producción como para los operarios).

D2: Diagnóstico de fallos/defectos y/o tratamiento de fallos/defectos. Acciones a ejecutar para determinar el origen del fallo o el origen del defecto. Pueden realizarse con la ayuda del operario.

\section{DISEÑO ESTRUCTURADO}

La principal idea asociada a la guía GEMMA que conviene matizar es que se trata de un enfoque de diseño estructurado (Fig. 2). Ante la complejidad de los factores que intervienen en la automatización de procesos, es conveniente utilizar el diseño estructurado con el fin de modelar, de forma parcial, las tareas. En el diseño estructurado de un sistema automatizado, aparecen tres módulos:

- Módulo de seguridad

- Módulo de modos de marcha

- Módulo de producción

La representación de la guía GEMMA tiene en cuenta la presencia de estos módulos, junto con las relaciones internas existentes entre módulos.

La jerarquía ilustrada en la Fig. 2, pretende intensificar la atención en el aspecto de seguridad de los sistemas automatizados en entornos productivos, como por ejemplo en situaciones de emergencia, en situaciones de fallos de dispositivos, o en situaciones de producción defectuosa, de forma que, ante estas contingencias, el módulo de seguridad es prioritario respecto a los otros módulos. En segundo lugar, es notoria la intervención del operario como parte integrante del sistema, pues aporta experiencia en el cambio de modo automático a modo manual cuando el funcionamiento del proceso lo requiere. Es decir, el control global del proceso puede ser debido a fragmentos de control intermitentes entre control automático y control manual. En tercer lugar, aparece el módulo de producción, que se entiende que está supeditado a los módulos precedentes, y en el que tenemos el funcionamiento de ejecución secuencial de activación y desactivación de estados mediante la lectura lógica de las transiciones.

Analizando estos módulos de forma inversa, la guía GEMMA pretende conservar la metodología de modelado GRAFCET, pero teniendo en cuenta que en el diseño estructurado de un sistema automatizado coexisten diversos GRAFCET parciales, los cuales hay que relacionar y jerarquizar convenientemente. Así, en el módulo de producción se concibe el organigrama de control básico en condiciones idóneas de funcionamiento, organigrama que se conoce como el GRAFCET de producción o GRAFCET de base; en el módulo de modos de marcha, el operario vigila el GRAFCET de producción y puede llegar a intervenir, si la situación lo requiere, mediante el cambio de control automático a manual (o viceversa, para reestablecer la situación) en este módulo, el organigrama se conoce como GRAFCET de conducción; finalmente, en el módulo de seguridad se procede al tratamiento de emergencias, fallos y defectos, con la posibilidad, por ejemplo, de que, se realice paradas de emergencias sobre el sistema, con las convenientes fases de solución de problemas y reconfiguración para volver a la condición de reinicio de la marcha y producción. En este último módulo, el organigrama se conoce como GRAFCET de seguridad. Uno de los factores importantes 
en el diseño estructurado aplicado a sistemas de automatización es la presencia del operario humano y la posibilidad de introducir decisiones sobre dispositivos a través del módulo de seguridad y el módulo de modos de marcha. Para clarificar la intervención del operario humano conviene diseñar un panel de mando que contemple las posibles decisiones humanas con los modos de la guía GEMMA (Rasmussen, 1986; Vicente, 1999; Ponsa y Díaz, 2006). Para el diseño del panel de mando se utilizan conceptos que aparecen en la normativa de seguridad en máquinas, así como especificaciones ergonómicas y el conjunto de situaciones a tratar mediante la guía GEMMA, tal como se desarrolla a continuación (Shneiderman, 1998; Ponsa y Díaz, 2007).

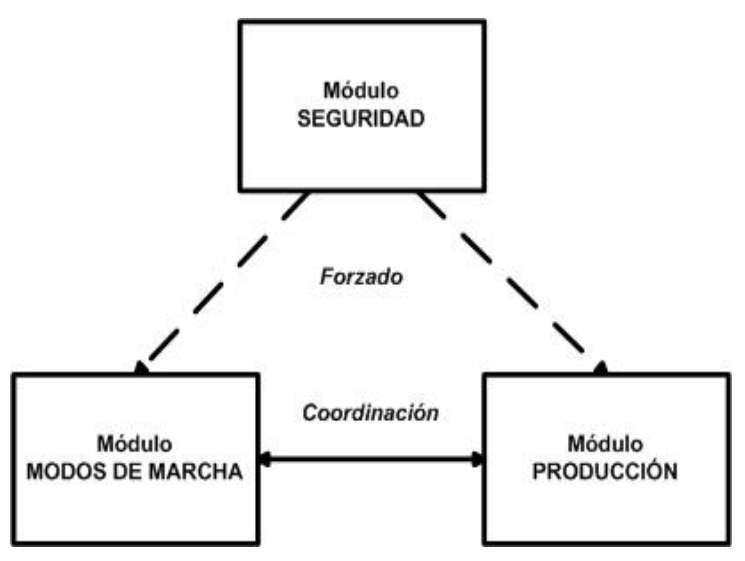

Fig. 2: Esquema de diseño estructurado

\section{DISEÑO DE PANEL INDUSTRIAL}

En automatización industrial existe una gran diversidad de dispositivos, que se engloban en lo que se conoce como interfaz persona-máquina, de manera que aquí no se pretende abordar el tema en detalle sino tan solo ofrecer un panel de mando apto para su uso con la guía GEMMA.

Los componentes elementales de un panel de mando (indicadores, pulsadores, selectores) cumplen con diversas especificaciones de seguridad en máquinas, como por ejemplo el grado de protección IP 54 o IP 65 (Nogareda, 2007).

Uno de los aspectos recogidos en estas normas, es la asociación del dispositivo con un color en concreto y una finalidad fijada. Así, un pulsador de marcha se representa mediante el color verde. El pulsador de paro convencional se representa mediante el color negro. Un pulsador de rearme, es decir cuando se suprimen las condiciones anormales y se reestablece el ciclo automático interrumpido, se representa mediante el color amarillo o azul. La parada de emergencia se representa con un dispositivo que se llama paro de emergencia y consta de una seta de color rojo dentro de un círculo amarillo, siendo de un tamaño netamente mayor al resto de pulsadores. El selector de posiciones se representa mediante el color negro con una pestaña en color blanco (Cavaría, 2007). En cuanto a los leds indicadores si se dispone de un led con luz blanca se asocia a una información general de servicio como por ejemplo la presencia de tensión en la máquina. Un led amarillo (ámbar) indica precaución. Se está ante una alarma que puede conducir a una situación peligrosa, como por ejemplo que se indique que la presión está fuera de los límites habituales de funcionamiento. El led verde indica que la máquina está preparada para entrar en funcionamiento. El led rojo indica una situación de fallo que requiere de la intervención inmediata del operario.

A partir de una guía ergonómica diseñada para interfaces industriales, se dispone a nivel vertical de tres niveles conceptuales sobre el panel de mando (Ponsa y Díaz, 2007). A la izquierda se representa la situación de servicio, en el centro, los dispositivos asociados a los modos de Marcha y Paro convencional, y finalmente a la derecha se disponen los dispositivos asociados a la Seguridad. Se ha preferido no situar el paro de emergencia en medio del resto de dispositivos y lo se ha situado 
abajo a la derecha para facilitar la accesibilidad sin ambigüedades. La Fig. 3, muestra la disposición final del panel de mando. En la parte de Servició se dispone un selector $(0,1)$ de manera que es el propio operario el que procede a poner en servicio la máquina o proceso; el led blanco indica esta acción. En la parte de Modos de Marcha/Paro el operario dispone de un selector de cuatro posiciones (MAN, 0, CaC, AUT). El modo 0 permite al operario detener la máquina en un instante concreto sin perjuicio del funcionamiento de la misma. La selección de 0 implicará que se desea parar la máquina al cabo de un instante, en contraste a $\mathrm{CaC}$ que significa el paro de la máquina al finalizar el presente ciclo. Las opciones MAN/AUT permiten el paso de control semiautomático a automático y viceversa. El selector se complementa con un pulsador de forma que realmente el operario primero selecciona y luego valida su decisión presionando el pulsador, evitando así situaciones accidentales que se podrían dar de manera fortuita. El led indicador de color verde informa del buen funcionamiento. En la parte de Seguridad se dispone del paro de emergencia y del pulsador de rearme para reestablecimiento del ciclo. Los leds indicadores mostrados informan de un posible fallo de un dispositivo (en este caso de un sensor, o de un actuador) y de una situación de alarma (en la que hay que estar precavidos ante un posible agotamiento de materia prima por ejemplo). Nótese que los leds quedan ordenados de izquierda a derecha como en un semáforo, verde, ámbar, rojo, facilitando que el operario asemeje este escenario a su representación mental, basada en el sentido común, y sea coherente con otras representaciones presentes en la sociedad.

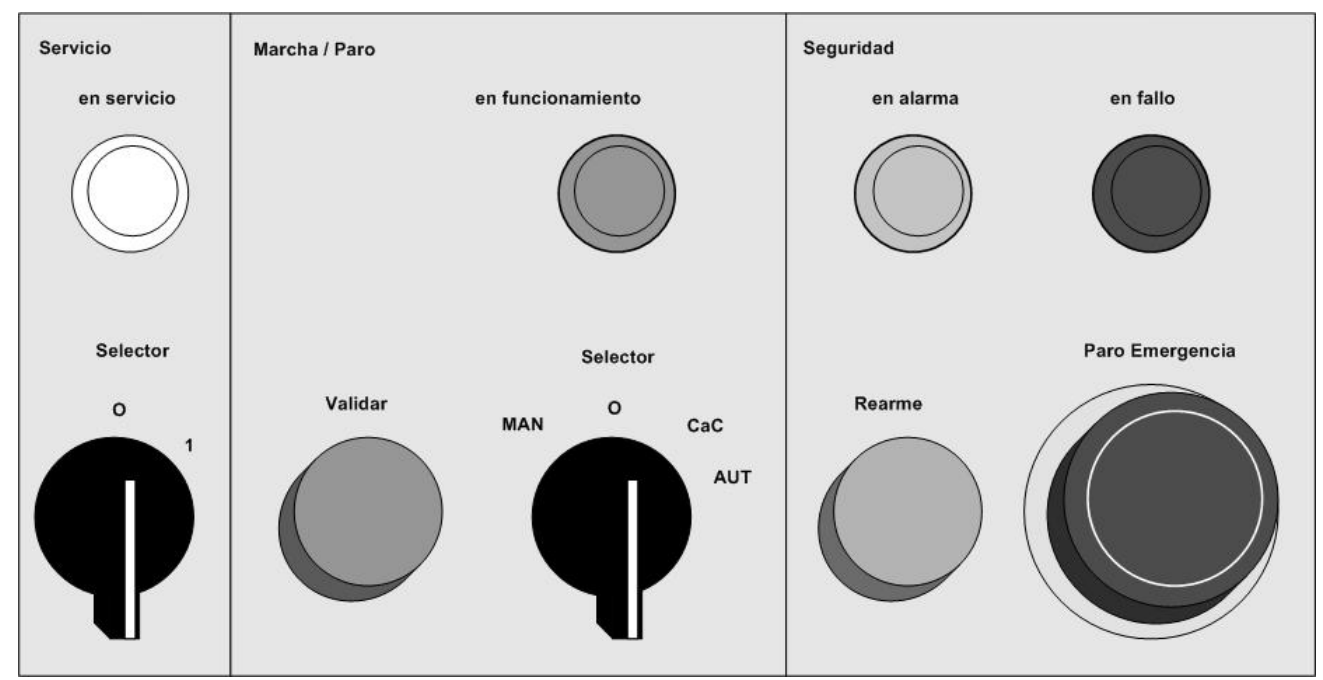

Fig. 3: Panel industrial necesario para la intervención del operario humano mediante el uso de la guía GEMMA

\section{SISTEMA DE FABRICACIÓN EDUCACIONAL}

El entrenamiento de estudiantes universitarios en el ámbito de la ingeniería técnica industrial mediante la aplicación de la guía GEMMA sobre sistemas de fabricación educacionales, permite validar la guía GEMMA como una metodología útil en la formación de futuros profesionales expertos en la automatización integral de procesos.

El centro docente Escola Politécnica Superior d'Enginyeria de Vilanova i la Geltrú, dispone de un sistema de fabricación flexible sobre el que realizar pruebas de aplicación de la guía GEMMA (Fig. 4) (Ponsa et al., 2005). Cada una de las estaciones dispone de un controlador lógico programable PLC por lo que se realizan tareas de automatización y supervisión (mediante programa "control supervisor y adquisición de datos" SCADA In TOUCH):

- Estación 1: alimentación, detección y distribución de piezas

- Estación 2: mecanización emulada y verificación de mecanizado

- Estación 3: Inspección y control de calidad de piezas mediante web cam

- Estación 4: Paletización o Ensamblaje de piezas mediante robot manipulador

- Estación 5: sistema rectangular cerrado de transporte con gestión de colas de espera en estaciones 


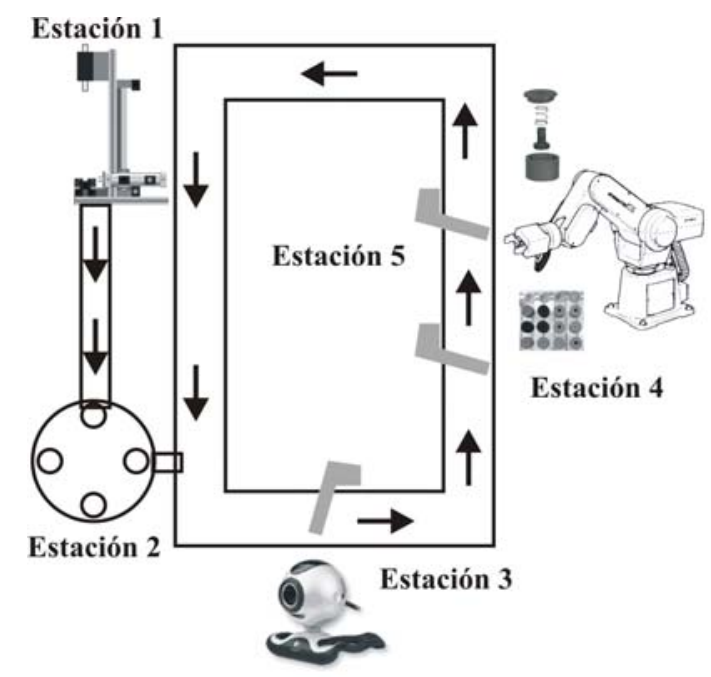

Fig. 4: Sistema de fabricación educacional

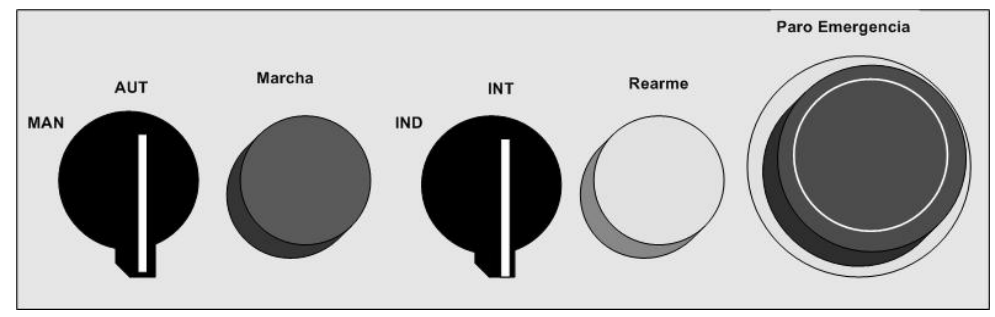

Fig. 5: Panel industrial suministrado por casa comercial de automatización

El sistema dispone de una red de comunicación Device Net y una red Profibus DP entre los controladores lógicos. La monitorización y supervisión se realiza mediante herramientas gráficas como LabVIEW o el SCADA InTouch. Los estudiantes de ingeniería técnica industrial, especialidad electrónica industrial, desarrollan diversas tareas para la puesta en marcha de la guía GEMMA sobre el sistema de fabricación educacional:

- Selección del controlador lógico

- Creación de la representación gráfica de la guía GEMMA y realización del GRAFCET de una estación

- Realización del código de programación del controlador

- Test del uso del panel de mando fabricado por el distribuidor comercial (véase Fig. 5), y test del uso de la guía GEMMA

- Propuesta de mejoras

Los estudiantes proceden a la intervención mediante el panel de mando de la Fig. 5. Para ello, se aborda la utilización de un panel de mando convencional diseñado previamente por una casa comercial para finalidad de uso didáctico con la estación ya comentada. Se comentan las ventajas e inconvenientes y la valoración hecha por los usuarios para la mejora del mismo. Las ventajas observadas en éste panel desde el punto de vista de la casa comercial son: bajo coste, posibilidad de trabajo individual o integrado entre estaciones y presencia de paro de emergencia. Las ventajas observadas en éste panel por parte de los ingenieros técnicos son: facilidad en el aprendizaje de su uso como interfaz, fácil conectividad física con el automatismo. Las desventajas constatadas por los ingenieros técnicos y recopiladas en el proyecto final de carrera llevado a cabo a lo largo de 6 meses son: dificultad de implementación de todas las situaciones contempladas en la guía GEMMA, y ausencia de leds indicadores informativos. Los ingenieros y estudiantes de ingeniería que han utilizado este panel presentan una serie de mejoras a incluir en el diseño de un futuro panel de mando que se adapte mejor a la cooperación entre operario-máquina (Fig. 6). A destacar: añadir leds informativos, añadir pulsadores para el accionamiento individual de actuadores neumáticos. 


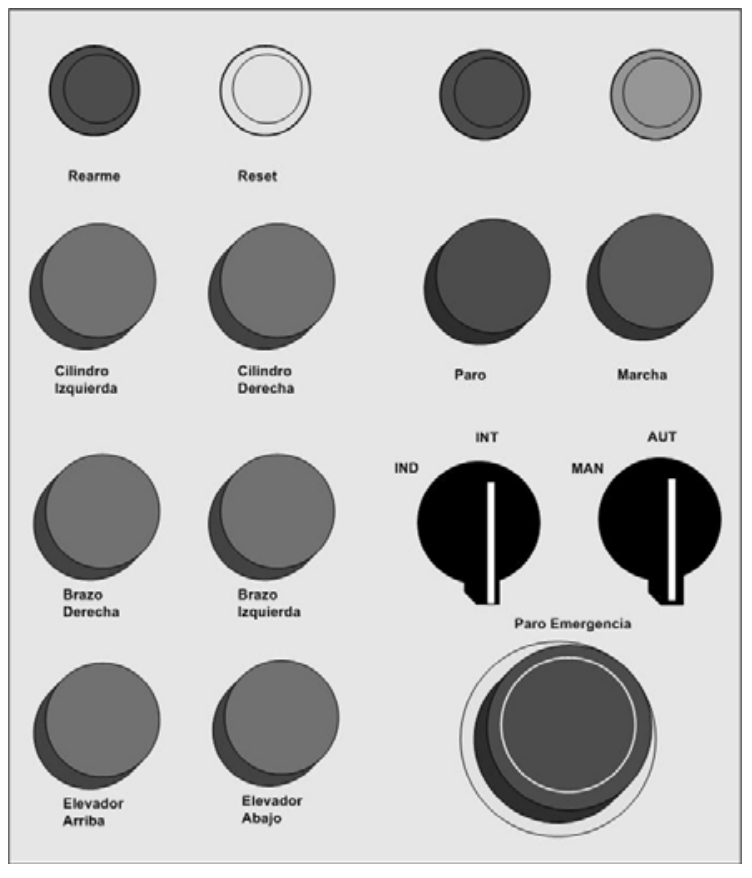

Fig. 6: Panel industrial diseñado por los estudiantes de ingeniería electrónica

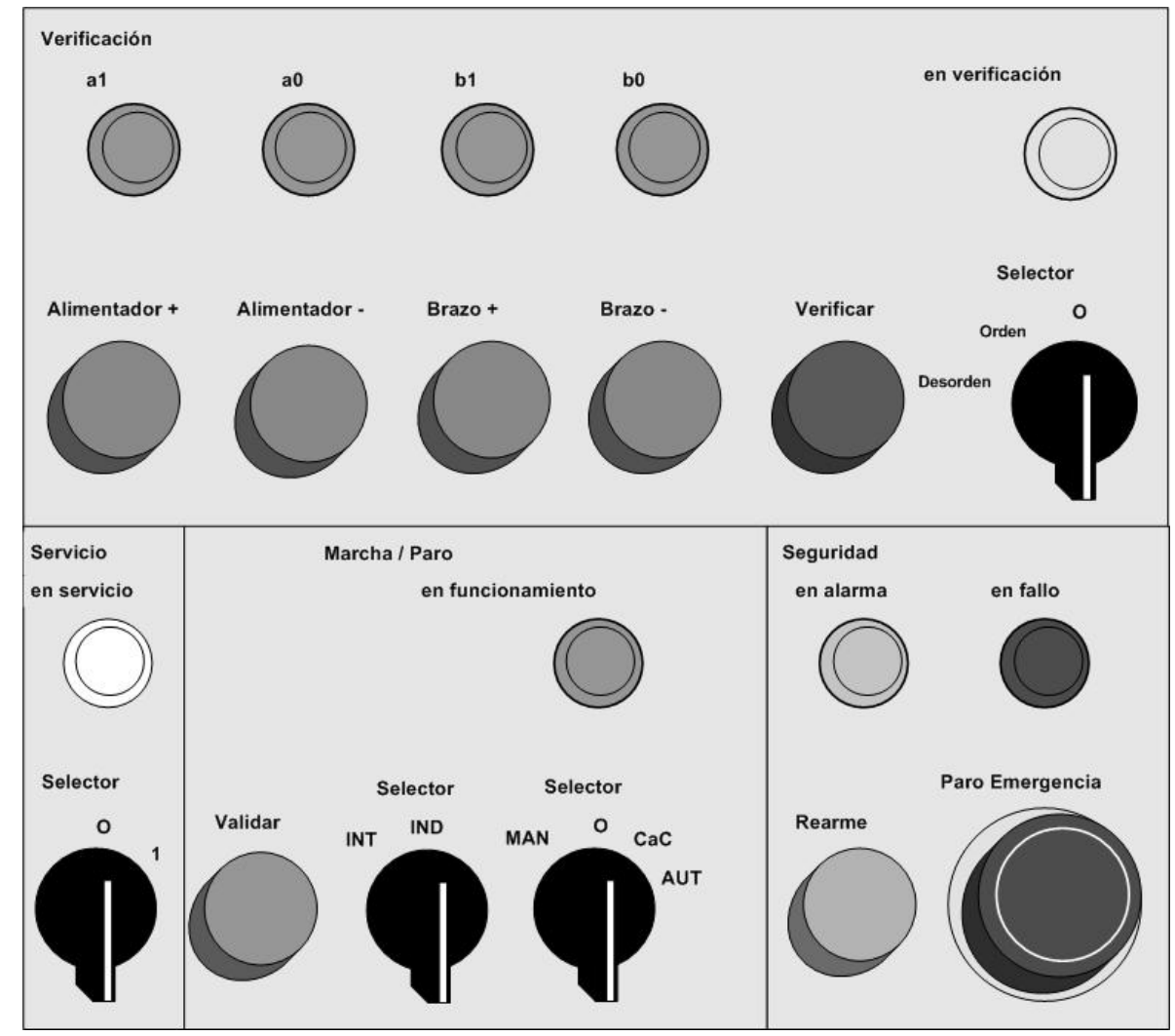

Fig. 7: Diseño ergonómico de panel industrial

En este sentido, los autores de este trabajo valoran las especificaciones de los usuarios y los criterios de diseño genéricos del apartado anterior para remodelar algunos aspectos concernientes a este panel de mando didáctico como:

- Debe suprimirse la ambigüedad en algunos pulsadores considerando una sola función para cada dispositivo. 
- Hay que incrementar notoriamente la presencia de dispositivos informativos visuales con la finalidad de informar claramente a los operarios de las posibles contingencias que puedan aparecer (finalización de tareas en una estación, el lote de piezas se acaba y hay que reponer material, etc.).

- En un escenario industrial el operario puede aportar su experiencia al diseñador del panel de mando para discutir la mejor disposición de pulsadores e indicadores de información.

- El panel de mando didáctico está situado sobre la estación y debería considerarse la posibilidad de situarlo de forma vertical para la mejora de la accesibilidad.

La versión definitiva del panel de mando se aprecia en la Fig. 7. A pesar del incremento de la complejidad del panel de mando respecto al representado en la Fig. 3, es conveniente indicar que en función de la tarea a realizar por parte del operario, la fase de Verificación es clave para prever incidencias en la máquina/proceso, de ahí que es preferible mejorar el mantenimiento preventivo y la seguridad del sistema persona-máquina al coste económico del panel de mando.

\section{CONCLUSIONES}

La guía GEMMA presenta una metodología integradora, concebida para clarificar el rol del operario humano en el ciclo de automatización de sistemas complejos, con aplicación en el control y supervisión de procesos industriales.

Los aspectos más destacables son:

- La guía GEMMA permite entrenar estudiantes de ingeniería en electrónica industrial como futuros operarios humanos

- Una aproximación multidisciplinar (estableciendo la relación entre control supervisor, automatización e interacción persona ordenador) es necesario para resolver sistemas complejos de automatización

- Es necesario enfatizar el diseño ergonómico del panel de mando y la incorporación del usuario (operario humano) en las fases iniciales del modelo de proceso de ingeniería en el ciclo de automatización

Como trabajos a realizar en un futuro cercano, es importante valorar los paneles diseñados para verificar la mejora de la eficiencia del sistema persona máquina.

Otro factor interesante a considerar, es la integración de la representación de la guía GEMMA en el marco de los sistemas SCADA tradicionales, y la integración de estos con otras herramientas de los sistemas productivos, como las herramientas de ejecución de la producción (Manufacturing Execution System MES) e incluso de gestión de la producción (Enterprise Resource Planning ERP).

\section{REFERENCIAS}

ADEPA ; "Le GEMMA. Guide d'Étude des Modes de Marches et d'Arrêts", Montrouge: ADEPA (1981) AFCET-ADEPA; "Le GRAFCET", Second edition Toulouse: Cépaduès. http://lurpa.enscachan/ grafcet.html (1995).

Armengol J., J. Melendez e J. Ayza; "Sistemas de supervisión”, Cuadernos CEA-IFAC, nº 1 (2000).

Cañas J.; "Personas y máquinas", Ediciones Pirámide, Colección "Psicología” (2004).

Català, A., P. Ponsa y L. Travé-Massuyès; "Artificial reasoners for human process supervision", Revista Iberoamericana de Inteligencia Artificial, no 9 Invierno /2000 (II/00), ISSN: 1137-3601, AEPIA, pag. 76-84 (2000).

Cavaría R.; "Equipo eléctrico de máquinas-herramientas. Órganos de servicio. Colores", Notas Técnicas de Prevención, NTP no 53, En URL: http://www.mtas.es/insht/ntp (2007).

Carver, L. y M. Turoff; "Human-computer interaction: the human and computer as a team in emergence management information systems“, en Emergency response information systems: emerging trends and technologies, 50(3) Marzo, ACM Press (2007). 
Garcia E.; "Automatizacion de procesos complejos", V Curso de Especializacion en Automática, Calpe, Alicante, Junio (2004).

Kontogiannis, T.; "Stress and operator decision making in coping with emergencies", Int. J. Human Computer Studies, 45 (1), 75-104 (1996).

Kontogiannis, T.; "Integration of task networks and cognitive user models using coloured Petri nets and its application to job design for safety and productivity“, Cognition, Technology and Work, vol. 7, 241-261, Springer-Verlag, November (2005).

Mondelo P.; "Ergonomía 3: Diseño del lugar de trabajo", Ediciones de la UPC, Aula Politècnica/ ETSEIB (2001).

Nogareda C.; "Mandos: ergonomía de diseño y accesibilidad", Notas Técnicas de Prevención, NTP nº 226, En URL: http://www.mtas.es/insht/ntp (2007).

Oncins M.; "Mandos y señales: ergonomía de percepción", Notas Técnicas de Prevención, NTP nº 241, En URL: http://www.mtas.es/insht/ntp (2007).

Petersen, J.; "Control situations in supervisory control", Cognition, Technology and Work, vol.6, 266274, Springer-Verlag, August (2004).

Ponsa, P. y A. Català. "Human supervision in industrial process", $7^{\text {th }}$ IEEE International Conference on Emerging Technologies and Factory Automation (ETFA'99), Barcelona, 18-21, october (1999).

Ponsa, P. y R. Vilanova; "Automatización de procesos mediante la guía GEMMA“, Ediciones UPC, Aula Politécnica/computación y control, nº 102, Noviembre (2005).

Ponsa, P., C. Raya y J.A. Soria; "A modelling and simulating method in educational manufacturing systems", Flexible Automation and Intelligent Manufacturing Congress, Bilbao (2005).

Ponsa P. y M. Díaz; "Microworld approach to supervisión activity modeling in industrial processes control". HCl related papers of Interacción 2004, R. Navarro y J. Lorés editores, Editorial Springer. (2006).

Ponsa, P. y M. Díaz; "Creation of an ergonomic guideline for supervisory control interface design", en Engineering Psychology and Cognitive Ergonomics, Edited by Don Harris, Lecture Notes Artificial Intelligence LNAI 4562, Editorial Springer (2007).

Rasmussen,J.; "Information processing and human interaction. An approach to cognitive engineering", Elsevier, Amsterdam (1986).

Shneiderman, B.; "Designing the user interface. Strategies for Effective Human-Computer Interaction". Third Edition, Addison-Wesley (1998).

Vicente, K.J.; "Cognitive work analysis“, Lawrence Erlbaum associates, publishers (1999). 